\title{
Ion acceleration in non-equilibrium plasmas driven by fast drifting electron
}

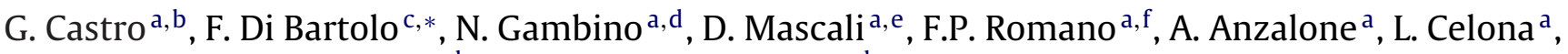

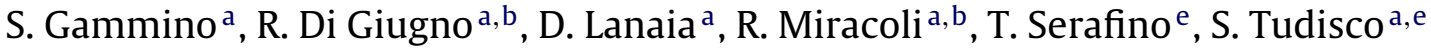 \\ a INFN- Laboratori Nazionali del Sud, via S.Sofia 62, 95123 Catania, Italy \\ b Università degli Studi di Catania, Dipartimento di Fisica e Astronomia, V. S.Sofia 64, 95123 Catania, Italy \\ c Università di Messina, V.le F. Stagno D'Alcontres 31, 98166, Messina, Italy \\ d Università degli Studi di Catania, Dipartimento di Metodologie Fisiche e Chimiche per L'ingegneria, Viale A.Doria 6, 95125 Catania, Italy \\ e CSFNSM, Viale A. Doria 6, 95125 Catania, Italy \\ ${ }^{f}$ CNR-IBAM Via Biblioteca 4, 95124 Catania, Italy
}

\section{A R T I C L E I N F O}

\section{Article history:}

Received 24 October 2011

Received in revised form 22 March 2012

Accepted 20 November 2012

Available online 4 January 2013

\section{Keywords:}

Electrostatic Bernstein waves

Plasma heating

Plasma vortex

Langmuir probe measurements

Multilayer plasma structure

Laser plasma

\begin{abstract}
A B S T R A C T
We hereby present results on ion acceleration mechanisms in non equilibrium plasmas generated by microwaves or high intensity laser pulses. Experiments point out that in magnetized plasmas X-B conversion takes place for under resonance values of the magnetic field, i.e. an electromagnetic mode is converted into an electrostatic wave. The strong self-generated electric field, of the order of $10^{7} \mathrm{~V} / \mathrm{m}$, causes a $E \times B$ drift which accelerates both ions and electrons, as it is evident by localized sputtering in the plasma chamber. These fields are similar (in magnitude) to the ones obtainable in laser generated plasmas at intensity of $10^{12} \mathrm{~W} / \mathrm{cm}^{2}$. In this latter case, we observe that the acceleration mechanism is driven by electrons drifting much faster than plasma bulk, thus generating an extremely strong electric field $\sim 10^{7} \mathrm{~V} / \mathrm{m}$. The two experiments confirm that ions acceleration at low energy is possible with table-top devices and following complementary techniques: i.e. by using microwave-driven (producing CW beams) plasmas, or non-equilibrium laser-driven plasmas (producing pulsed beams). Possible applications involve ion implantation, materials surface modifications, ion beam assisted lithography, etc.
\end{abstract}

(C) 2012 Elsevier B.V. All rights reserved.

\section{Introduction}

The need of intense beams of ions at moderate energy for industrial or scientific applications is constantly growing. At the same time, the exceptional improving in plasma physics understanding, including non-linear mechanisms in laser plasma interaction, has provided absolutely new methods for particles acceleration. In laser-plasma-based ion acceleration, the energy scales with the laser intensity; in the case of table-top lasers operating at moderate intensities $\left(I<10^{12} \mathrm{~W} / \mathrm{cm}^{2}\right)$ and in a nanosecond domain ions are typically accelerated up to some keV. Laser Produced Plasmas (LPP) are employed in several fields of matter and nuclear physics, such as thin film deposition, ion acceleration and nuclear fusion [1,2]. In a low energy domain, like that of laser plasmas produced in irradiance regime of $10^{12} \mathrm{~W} / \mathrm{cm}^{2}$, the density is very low (on the order of $10^{19}-10^{21} \mathrm{~cm}^{-3}$ ) if compared with stars, but temperature and density can be rescaled to simulate phenomena of astrophysical interest.

\footnotetext{
* Corresponding author.

E-mail addresses: fdibartolo@unime.it, fdibartolo@Ins.infn.it (F. Di Bartolo).
}

Spontaneous formation of a high-speed plasma flow has been recently observed at LNS when the plasma was ignited through non-linear microwave plasma interaction. Microwave ignited plasmas are largely employed as powerful injectors for particles accelerators or like high intensity proton sources and in industrial applications. The plasma ignition is usually obtained by means of microwaves at $2.45 \mathrm{GHz}[3,4]$, axially launched inside the plasma chamber, where a strongly non uniform magnetostatic field exists (with $0.1 \mathrm{~T}$ of maximum value). If the wave is properly launched, in under-resonance regions the $\mathrm{X}-\mathrm{B}$ conversion is possible [5], i.e. the incoming electromagnetic extraordinary mode is converted into an electrostatic Bernstein wave. Signs of BW generation are the transition from an underdense to overdense plasma state, and specially the appearance of high energy electrons. In the new setup designed at LNS clear signatures of BW generation have been observed. During BW heating regime, the plasma transits from an equilibrium to a strongly non-equilibrium regime governed by turbulence. The strong electric field associated with the nascent EBW drives $E \times B$ drifts causing ion and electron acceleration.

We also designed a novel setup to study nuclear astrophysics by employing LPP [6]. By studying the evolution of single expanding plasma, we observed, other than the classical hydrodynamics expansion, some non linear processes driven by the formation of 


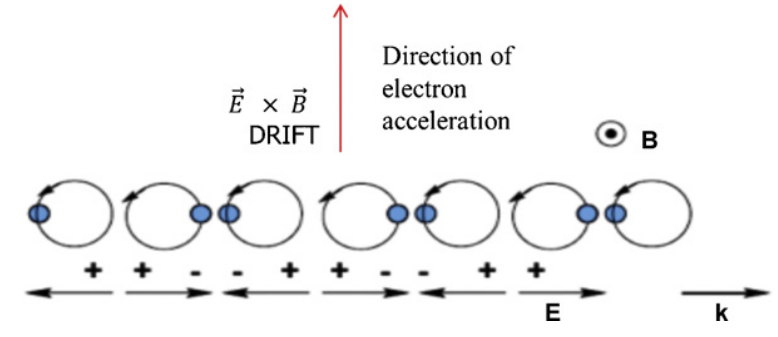

Fig. 1. Description of the charge bunching creating the EBW waves and direction of $E \times B$ drift

Double or Multi-layers. Mechanisms of ion acceleration have been observed, based on the "blow-off" of prompt electrons which in turn drive instabilities, producing finally the Coulomb-like explosion and a multi layered plasma bulk. Similar electric fields have been found in microwave driven and laser driven plasmas at intensity of $10^{12} \mathrm{~W} / \mathrm{cm}^{2}$.

\section{Theory of $X-B$ conversion mechanism}

ECR plasmas are density limited [7], in facts the electromagnetic waves cannot propagate above the so-called cut-off density: $n_{\text {cut-off }}=\left(\varepsilon_{0} m_{e} / e^{2}\right) \omega^{2}$. Electron Bernstein Waves (EBW) [8] are able to propagate in overdense plasmas, and thus they are an option to increase the density over the cut-off limit $[7,9]$. They are crucial for plasma ignition in nuclear fusion experiments, and represent a promising technique to improve the performances of existing plasma-based ion sources, which are critically dependent on the plasma density. EBW are generated at Upper Hybrid frequency (UHR) $[9,10]$, i.e. the resonance of $X$ wave, where the condition: $\omega_{\mathrm{RF}}=\sqrt{\omega_{p}^{2}+\omega_{c}^{2}}$ is valid, being $\omega_{c}$ the cyclotron frequency and $\omega_{p}$ the plasma frequency, and propagate perpendicularly to the magnetic field as layers of electron density accumulation and rarefaction sustained by the cyclotron motion (Fig. 1).

Evidence of the conversion from Electromagnetic to Electrostatic waves is given by parametric decay, which can be visualized by means of the presence of sidebands (due to the creation of ion waves in the process) in the E.M. spectrum at UHR position. Dispersion relation of the Bernstein waves [11] is:

$1-\left(\frac{k_{B} v_{\text {th }}}{\omega_{p}}\right)^{2}=e^{-k_{B}^{2} r_{L}^{2}} I_{0}\left(k_{B}^{2} r_{L}^{2}\right)-\left(\frac{\omega}{\omega_{c}}\right) \frac{\sum_{q} e^{-k_{B}^{2} r_{L}^{2} I_{q}\left(k_{B}^{2} r_{L}^{2}\right)}}{q^{2}-\frac{\omega}{\omega_{c}^{2}}}$

where $k_{B}$ is the wave number of the Bernstein wave, $v_{\text {th }}$ is the electron thermal velocity, $r_{L}$ is the electron Larmor radius, $\omega_{p}$ and $\omega_{c}$ are plasma and cyclotron frequencies, $\omega$ is the pumping frequency, $I_{q}$ is the Bessel function, $q=1,2, \ldots, N$. BWs exhibit a resonance when the denominator of equation 1 vanishes, i.e. when:

$B_{\text {resonance }}=\frac{1}{q}\left(\frac{m}{e} \omega_{\mathrm{RF}}\right)=\frac{1}{q} B_{\mathrm{ECR}} \quad q=1,2, \ldots N$.

corresponding to cyclotron harmonics. The absorption mechanism can be explained by the Segdeev-Shapiro damping model [11]. The maximum energy available by the electron $W_{\mathrm{MAX}}$ depends by the electric field $E$ of the Bernstein waves and by the magnetic field $B$ in the point where absorption takes place [12]:

$W_{\mathrm{MAX}}=\frac{1}{2} m_{e}\left(\frac{E}{B}\right)^{2}$

where $m_{e}$ is the electron mass. It can be shown [12] that if the magnetic field is directed along the $z$ axis and the wave propagates along the $x$ axis, the electrons are accelerated along the $y$ axis because of the $\vec{E} \times \vec{B}$ drift acting on electrons. In a cylindrical symmetry these

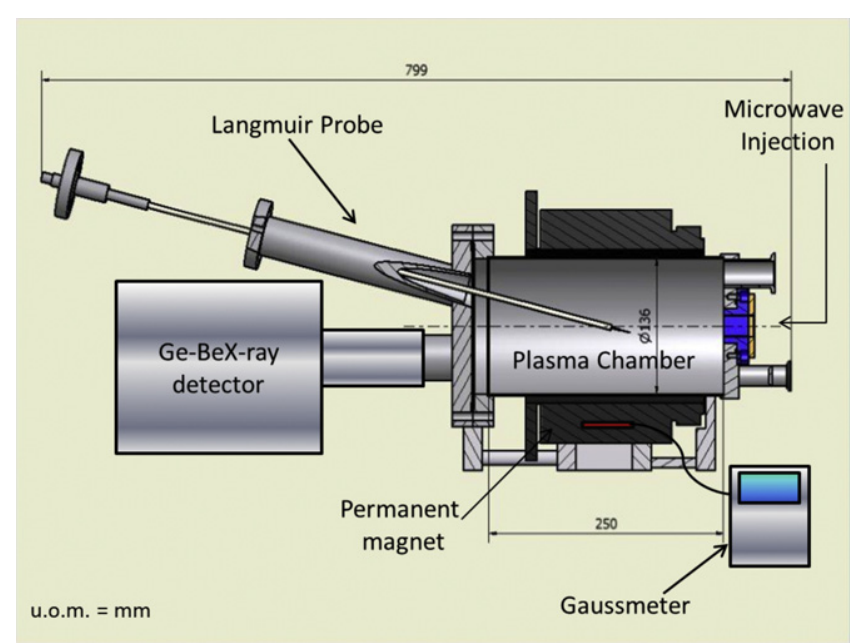

Fig. 2. Render view of experimental set-up.

electrons will be accelerated into concentric circles, creating an azimuthal flow rotating around the plasma chamber axis. Experimental results [13] show that also a radial component of motion can arise because of a $\vec{F} \times \vec{B}$ drift, where $F$ is a friction force due to the collisions. The result is the formation of a "typhoon-shaped" plasma vortex $[13,14]$. Thus EBW-heated Plasma can be represented as made of a series of vortex-like energetic electron layers at the cyclotron harmonics positions (where EBW are absorbed) separate by bulk plasma regions.

\section{Experimental setups}

Parts of the tests have been carried out in a small-size plasma reactor (a stainless-steel cylinder $24 \mathrm{~cm}$ long, $14 \mathrm{~cm}$ diameter) that is essentially a Microwave Discharge Ion Source usually working at $2.45 \mathrm{GHz}$. A NdFeB permanent magnets system generates an offresonance magnetic field along the plasma chamber axis (with a maximum of $0.1 \mathrm{~T}$ on axis). Microwaves have been generated using a Traveling Wave Tube (TWT) working in the range $3.2-4.9 \mathrm{GHz}$. Most of the experimental results were collected at $3.7478 \mathrm{GHz}$. Nitrogen has been flowed inside the reactor, keeping constant the pressure at $10^{-4}$ mbar. The microwaves were axially launched inside the chamber and, due to the formation of standing waves, extraordinary waves (with non parallel $\vec{k}$ vectors with respect to the $B$ axis) can be generated [15]. The measurements of plasma temperature and density have been carried out using a movable Langmuir Probe (LP). The same system can host a small wire used as a local electromagnetic antenna, which can be connected to a Spectrum Analyzer (SA) for the plasma spectral emission analysis. A Ge-Be X-ray detector has been used for the measurement of Xrays spectra. The detector was placed in the extraction flange and it was separated by the plasma only by a $75 \mu \mathrm{m}$ thick kapton layer, in order to allow the detection of $\mathrm{X}$ rays in the energy range 1-30 keV. A CCD camera has been used to visualize plasma structure within the chamber at different powers. A gaussmeter enabled us to measure variations of magnetic field linked to internal currents in the plasma. A render view of the experimental set-up is shown in Fig. 2.

The experimental set-up used to characterize the laser produced plasmas is shown in Fig. 3 and is fully described in [16,17]. It consists of a compact cylindrical stainless steel vacuum chamber, having a diameter of $250 \mathrm{~mm}$ and being $240 \mathrm{~mm}$ high. Four DN40 flanges are located at $90^{\circ}$ with respect to the chamber axis, while additional DN25 and DN16 form angles going from $15^{\circ}$ to $45^{\circ}$. The plate where the chamber is located was designed and made at LNS and has lateral dimensions of $600 \mathrm{~mm}$ The laser is a Quanta 


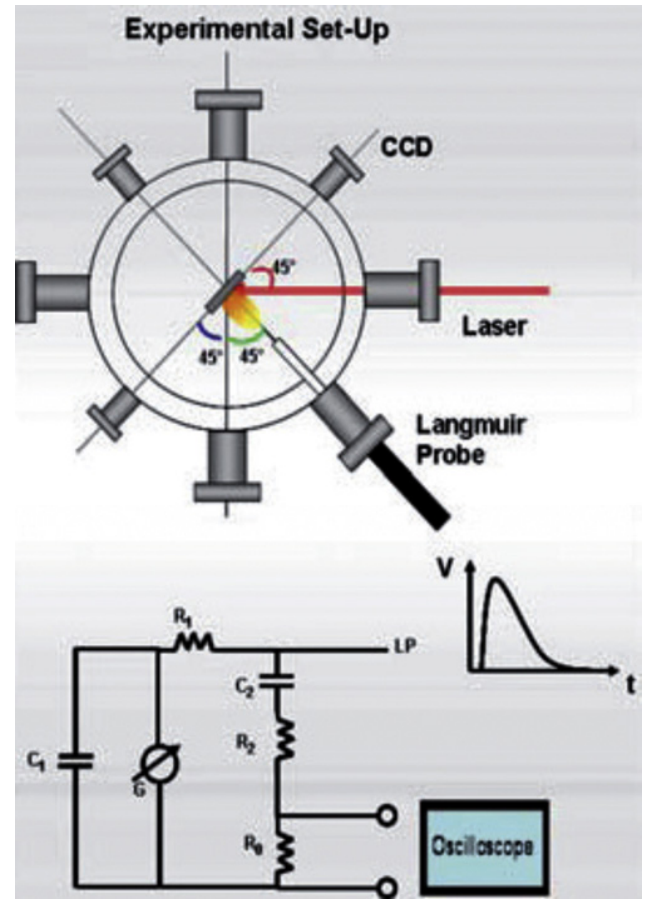

Fig. 3. Experimental set-up: internal view of the chamber with the Langmuir Probe close to the target surface and RC read out circuit.

system Nd-YAG operating at a power irradiance of $10^{12} \mathrm{~W} / \mathrm{cm}^{2}$, with $6 \mathrm{~ns}$ of pulse duration and FHWM of $1064 \mathrm{~nm}$ focused on a $\mathrm{Al}$ target through a $250 \mathrm{~mm}$ focal length lens, obtaining spot size on the order of $200 \mu \mathrm{m}$. For the determination of the plasma parameters the same movable LP described in the previous experimental set-up was used (a tungsten $5 \mathrm{~mm}$ long cylindrical thin tip, with a diameter of $0.15 \mathrm{~mm}$, connected to a rigid $300 \mathrm{~mm}$ long coaxial cable, having a thickness of $3 \mathrm{~mm}$ ). The signal was read out with the RC circuit shown in the inset of Fig. 3 permitting us to know exactly the current value (on the order of several of hundred $\mathrm{mA}$ ) flowing through the probe, fundamental to estimate the electron plasma density. The unbiased probe was placed at different distances from the target: from $2.5 \mathrm{~mm}$ to $50 \mathrm{~mm}$ with spatial steps of few $\mathrm{mm}$. When $V=0$ the probe collects both electrons and ions: ions hit the probe attracted by a potential which is given by $V-V_{p}=-V_{p}$, where $V_{p}$ is the plasma potential. The electrons are partially repelled whereas the ions are attracted. Hence the LP signal can be interpreted as follows: if $J_{i}>J_{e}$, the number of collected ions is larger than the electrons one, otherwise the probe shows a negative signal. Usually the ions dominate at such repelling voltages $\left(V_{p}\right.$ is typically around $30-80 \mathrm{~V}$, this means that electrons are repelled by a large negative potential). Therefore the total probe current at intermediate positive or small negative voltages will be $I(V)_{\text {tot }}=I_{i}+I_{e}$, where $I_{i}$ and $I_{e}$ are respectively the ion and the electron current collected by the probe.

\section{Results and discussion}

\subsection{Plasma vortex formation in EBW-heating regime}

At $3.7478 \mathrm{GHz}$ microwave frequency the Electron Cyclotron Resonance (ECR) cannot occur at all, thus prohibiting direct electromagnetic wave absorption at the resonance. Therefore the strong plasma heating observed through X-ray spectroscopy (see Fig. 4) is the first confirmation of EBW damping at higher cyclotron harmonics (the second one is placed inside the plasma chamber). A second signature of EM to ES waves conversion is given by

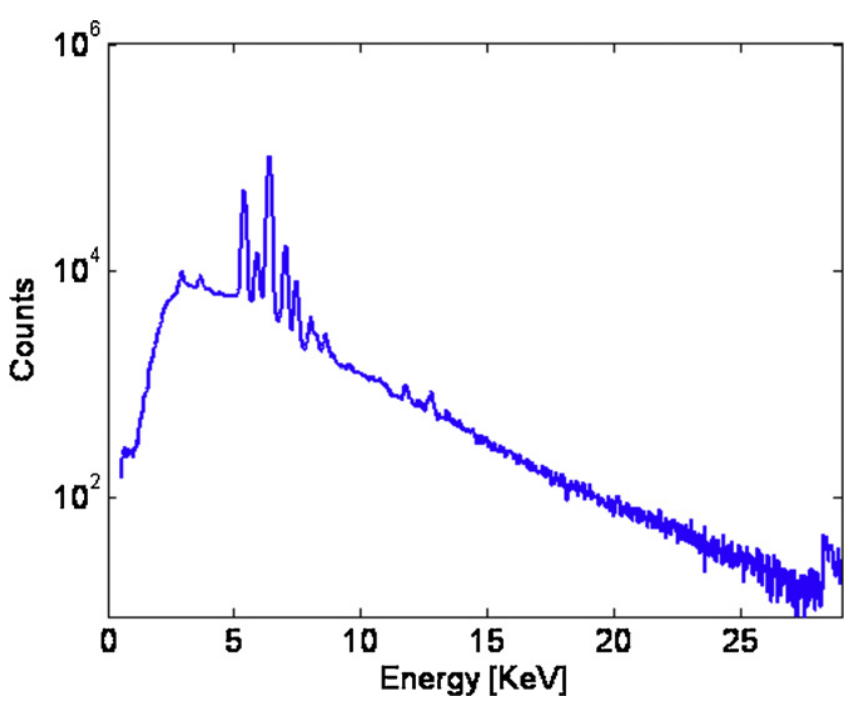

Fig. 4. X-rays spectrum emitted by the EBW-sustained plasma revealed at $3.7478 \mathrm{GHz}, 100 \mathrm{~W}$ pumping power and $9 \times 10^{-5}$ mbar pressure.

sidebands detected in the EM spectrum in the range of $\mathrm{KHz}$ (see Fig. 5). The measurements of X-rays energy shown in Fig. 4 provide spectral temperatures in the range 1-4 keV: in particular we found $T=1.5 \mathrm{keV}$ below $80 \mathrm{~W}$; at $80 \mathrm{~W}$ a threshold exists and spectral temperature grows up to $4 \mathrm{keV}$. Endpoint energies allow us determine the maximum energy that an electron has gained. Values up to $40 \mathrm{keV}$ have been measured. Such values are incompatible with direct EM absorption at ECR (classical ECR heating) in unconfined plasmas, whose typical temperatures are of the order of tens or hundreds eV.

Electric field of EBW is related to the endpoint electron energy derivable from X-rays spectra through the Segdeev and Shapiro approach. From Eq. (3) it comes out that:

$E_{\mathrm{EBW}}=B \sqrt{\frac{2 W_{\mathrm{MAX}}}{m_{e}}}$

By setting $B=0.1 \mathrm{~T}$ and $W_{\mathrm{MAX}}=40 \mathrm{keV}$, one obtains an electric field of $1.2 \times 10^{7} \mathrm{~V} / \mathrm{m}$. The aforementioned threshold in pumping wave power corresponds to a marked change in plasma configuration observable in the visible range: it is useful to give a look to the pictures taken by means of a CCD and displayed in Fig. 6 . At low power plasma assumes the shape of a bright ring; at $P=80 \mathrm{~W}$ the plasma modifies suddenly its shape and a dark hole surrounded by a high-brightness area becomes visible. The change of configuration causes the increase of the X-ray spectral temperature. It seems clear

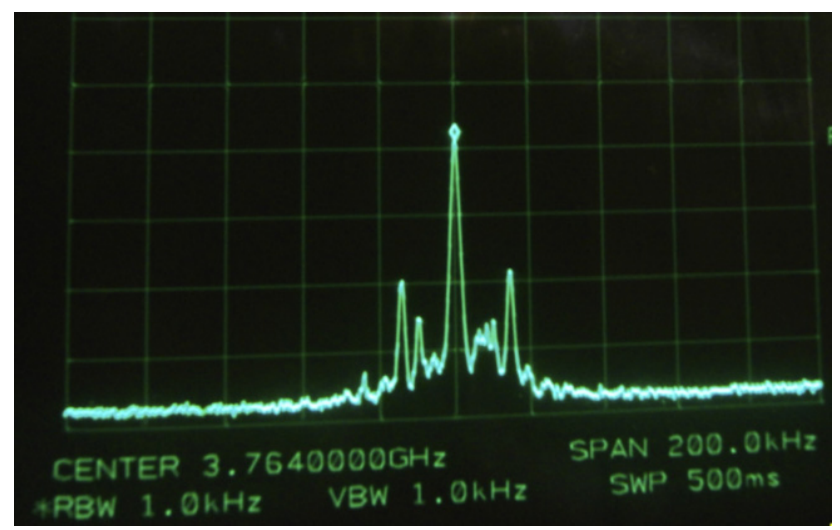

Fig. 5. Electromagnetic spectrum emitted by the EBW-sustained plasma during EM to ES conversion. 

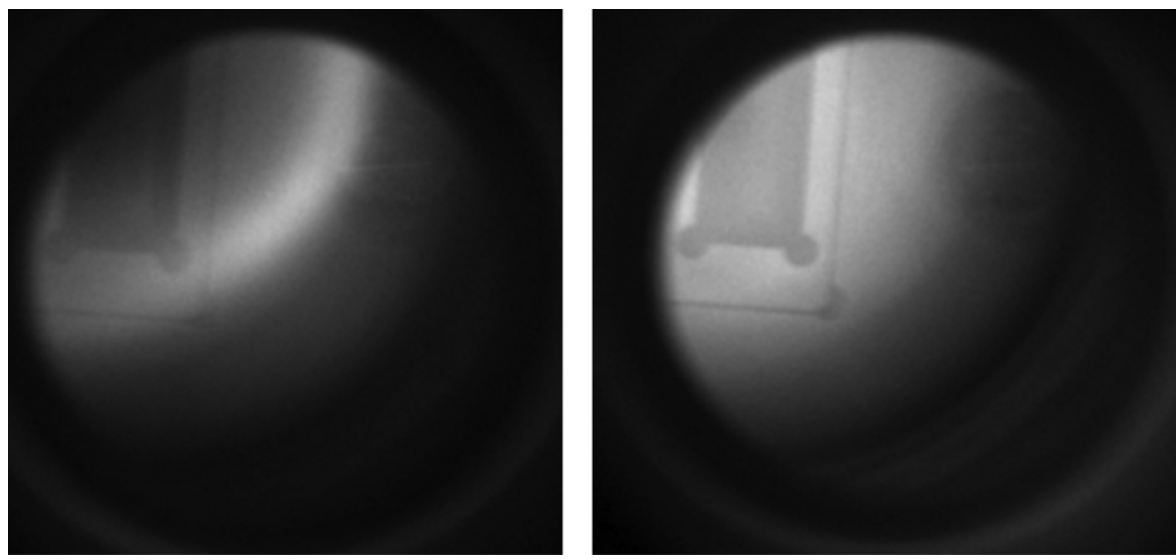

Fig. 6. Images of the EBW-sustained plasma at $f=3.7478 \mathrm{GHz}$ and Pressure $=1.5 \times 10^{-4} \mathrm{mbar}$ (on the left Microwave power is $50 \mathrm{~W}$, on the right is $250 \mathrm{~W}$ ).

that the plasma goes through two metastable equilibrium configurations, each one characterized by a typical plasma shape and X-ray energy.

The measurements carried out by means of the gaussmeter show clearly the presence of internal current inside the plasma. An additional azimuthal magnetic field appears promptly after the plasma ignition (see Fig. 7). The azimuthal magnetic field is generated by an axial plasma current directs toward the microwave window. In this power range no other variation of magnetic field are revealed. Increasing the pumping wave power, a further additional magnetic field component arises along the chamber axis, and this is a signature that an inner plasma azimuthal current has been generated. Moreover, at this threshold the azimuthal magnetic field decreases suddenly and then increases again for higher microwave power. The plasma state transition is additionally accompanied by a strong decrease of the downstream (with respect to the microwave window) neutrals pressure. This is a surprising and a not conventional mechanism for classical ECR-heated plasmas (Fig. 8). The threshold could be probably explained as the transition from a collisionless to a collisional plasma regime. EBWs, in fact, enable to reach electron density up to $2 \times 10^{12} \mathrm{~cm}^{-3}[18]$. At such densities neutral and ions are no more able to cross the rotating ring, and thus they are carried away from the high energy drifting electron flux, being ionized. Since neutrals are pumped inside the chamber from a hole placed close to the microwave window, they cannot flow downstream since promptly ionized when passing through the ring so that the measured pressure decreases as soon as plasma hole

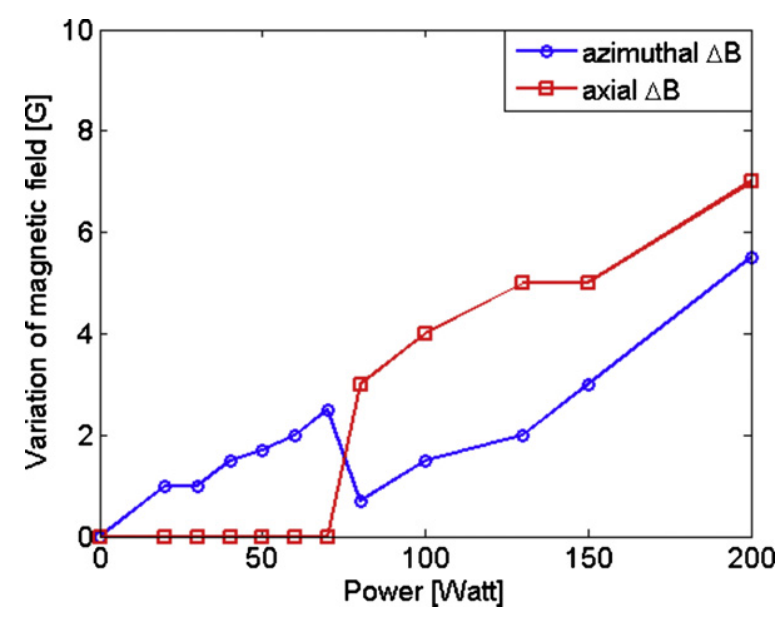

Fig. 7. Variation of axial and azimuthal magnetic field when microwave power is increased. A threshold is visible at $80 \mathrm{~W}$. appears. Furthermore, because ions cannot reach the same velocity of drifting electrons (due to conservation of linear momentum) a resulting azimuthal current arises to preserve quasi-neutrality in the plasma hole. The flow pattern is very similar to a monopole vortical structure, with a sink at the center. The expulsion of electrons and ions by the hole causes the axial current, which we indirectly measured through magnetic field variations.

The fingerprint of self-generated plasma fluxes in case of EBWheating regime is given by the damage visible on the walls of the plasma chamber, as displayed in Fig. 9a. Erosion of the stainless steel corresponds to positions where plasma rings are formed, and it is due to the sputtering given by high energy ions. In order to have a first check of the presence of the axial current directed toward the microwave window (as indicated by magnetic measurements), we covered the window with a kapton layer. The layer has been heavily damaged (a hole was formed after few seconds of operations) only in the central region, corresponding to the axis of the chamber, as visible in Fig. 9b.

\subsection{Self acceleration of ions in a fast-expanding LPP}

The set of measurements, carried out with unbiased LP placed along the expansion axis of a Al laser produced plasma, permitted us to investigate the plume dynamics without perturbing excessively its expansion. In Fig. 10 the time of flight signals for different $d$ (where $d$ is the Langmuir Probe - Target surface distance) are reported. At $d=2.5 \mathrm{~mm}$ we revealed a negative peak $10 \mathrm{~ns}$ after the

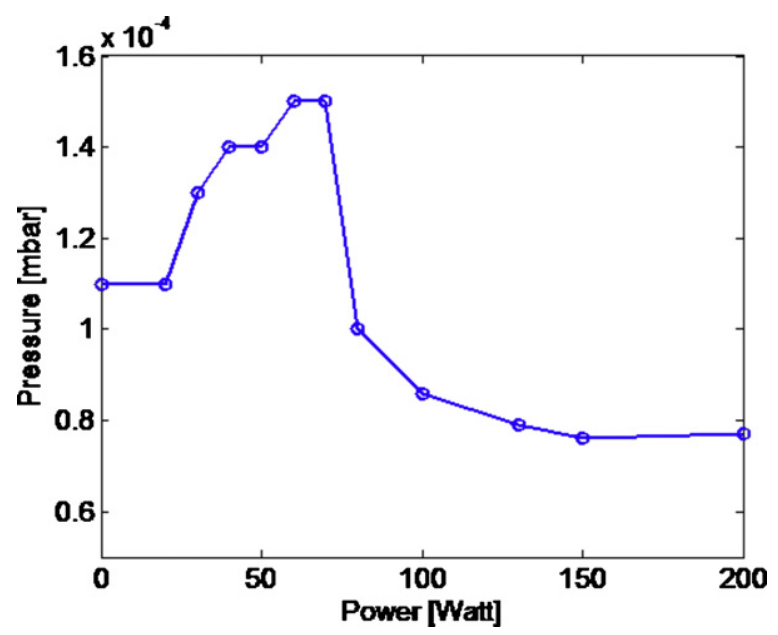

Fig. 8. Variation of neutral pressure when microwave power is increased. A threshold is visible at $80 \mathrm{~W}$. 


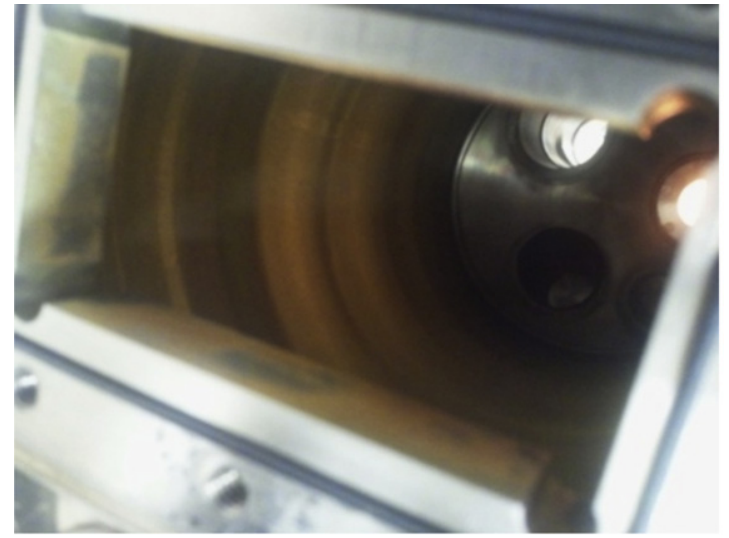

a

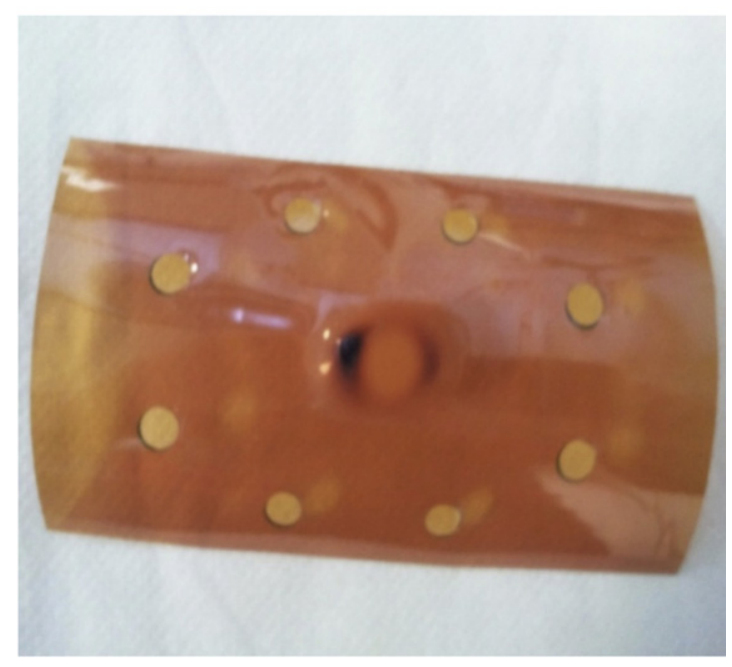

b

Fig. 9. (a) Damages to the plasma chamber walls due to fast rotating ions inside the EBW-sustained plasma. (b) Damages to a kapton layer positioned along the chamber axis due to axial current.

photopeak starting signal. This negative signal is due to fast escaping electrons. The fast electron peak is then followed by several positive ion peaks. After $150 \mathrm{~ns}$, the bulk plasma expansion follows, with the expected dropping density. At 400 ns a hot electron tail, which is expanding quite slowly, impacts on the probe. This dynamics is favored by the high three body recombination rate, which permits that hot electrons survive in the plasma at longer expansion times [17]. The multi-peak are also detected for $d=4.5 \mathrm{~mm}$ and $d=6.5 \mathrm{~mm}$ and disappear at $d=14.5 \mathrm{~mm}$, since at larger distances

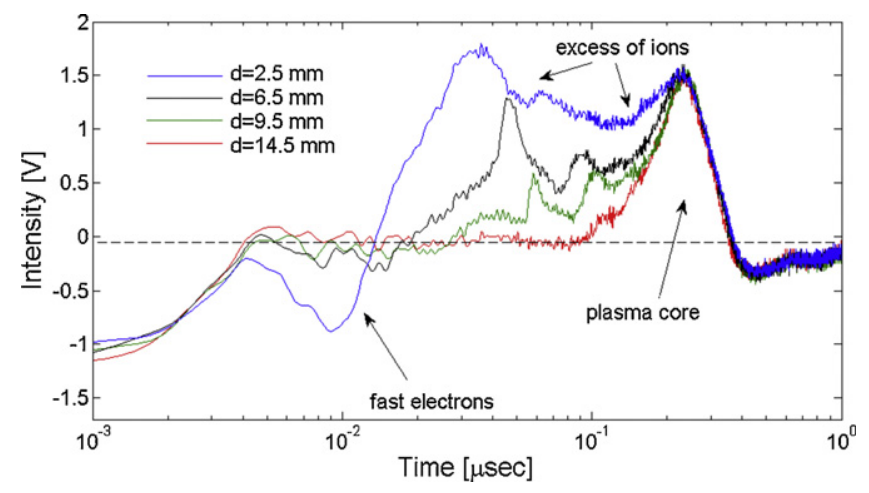

Fig. 10. Time of flight signals vs. time for $V_{p}=0 \mathrm{~V}$ and different probe positions.

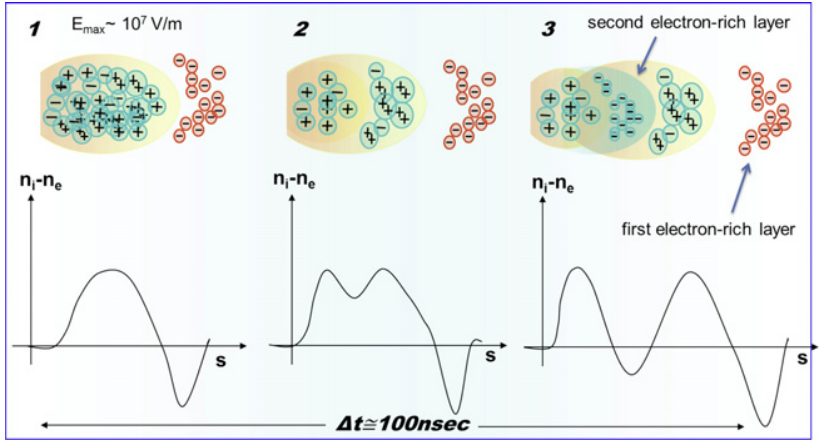

Fig. 11. Representation of the mechanism of multi-layer formation. Ion and electron density were reported as a function of the distance from the target.

the transverse area of the probe is too small to detect such particles. The first $100 \mathrm{~ns}$ of the plume expansion are therefore characterized by a multiplicity of non neutral layers, which we can define as Multi-Layers (ML). These data are similar to the ones reported by Bulgakova et al. [19]: they observed strongly non-linear dynamics leading to quasineutrality violation in high laser fluence regimes. The fast escaping of electrons triggers these non-linearities in the expansion of the bulk plasma. The latter partially fragments in a multiplicity of non-neutral layers, each one populated by ions at given charge state. An estimation of the kinetic energy of the first layers of fast ions, evaluated from the LP-TOF signals, gives energies on the order of $1-4.5 \mathrm{KeV}$. This self-acceleration process takes place in a timescale on the order of hundred of nanoseconds. Numerical calculations also show that the electric field developing during the ML expansion has a maximum value on the order of $3 \times 10^{7} \mathrm{~V} / \mathrm{m}$ [16].

The mechanism of plume fragmentation and non-linear ion acceleration can be depicted as follows (see Fig. 11). Initially fast electrons develop a double layer (DL) in the plasma core that accelerates the ions and splits the different charge states. The splitting produces bunches separated by some Debye lengths and electrons diffuse in the inter-bunches space accelerating also the lower charge states bunches. Therefore, a plurality of charged layers is formed. By analyzing the $I-V$ curves collected through the LP we found a two electron temperature (TET) plasma when the probe was placed at $d=4.5 \mathrm{~mm}$. TET plasmas [16], as predicted by several theories, are good candidate for developing instabilities and multifragmentation, as predicted, for example, by Hora [20].

\section{Conclusions}

The role of inner self-generated electric fields for ion acceleration is attracting a growing interest in the accelerator expert community, since they could allow to realize table-top machines available for a multiplicity of applications. In the keV range, ions accelerated in small-size plasma reactors or by means of small-size lasers could be employed for a variety of technological processes, from material surface treatment to ion implantation. We have demonstrated that ions can be accelerated in case of strong electric field induced by intense laser pulses, while different experimental evidences show that ion acceleration takes place also in plasma excited by means of electrostatic waves. In both cases ion acceleration is driven by fast drifting electrons and estimated electric fields are of the order of $10^{7} \mathrm{~V} / \mathrm{m}$. In laser produced plasmas, ion energies range from 1 to $4 \mathrm{keV}$. Further experiments will be needed for a more precise measure of ion energy especially in case of the plasma reactor. 


\section{Acknowledgments}

The support of the NTA-Helios Strategic Project of INFN and of the 5th National Committee is gratefully acknowledged. The authors are grateful to L. Neri, G. Cosentino, A. Musumarra and D. Santonocito for their support during experimental activity. The cooperation of L. Allegra and F. Chines has been essential for the present work. We acknowledge the financial support of the European Union Seventh Framework Programme FP7/2007- 2013 under grant agreement no. 262010 - ENSAR. The EC is not liable for any use that can be made on the information contained herein.

\section{References}

[1] J. Nuckolls, et al., Nature 239 (1972).

[2] T. Tajima, et al., Physical Review Letters 43 (1979).

[3] T. Taylor, J.S.C. Wills, Nuclear Instruments and Methods A 309 (1991) 37.

[4] G. Ciavola, S. Gammino, G. Raia, J. Sura, Review of Scientific Instruments 65 (1994) 1110.

[5] H.P. Laqua, Plasma Physics and Controlled Fusion 49 (R1) (2007).
[6] D. Mascali et al., Proc. IAU workshop, Giardini-Naxos (2010) Advances in Plasma Astrophysics, Proceedings IAU Symposium No. 274, 2010 , http://dx.doi.org/10.1017/S1743921311006557

[7] Y.Y. Podoba, H.P. Laqua, G.B. Warr, M. Schubert, M. Otte, S. Marsen, F. Wagner, Physical Review Letters 98 (2007) 255003.

[8] I.B. Bernstein, Physical Review 109 (1958) 10

[9] J. Preinhaelter, et al., Plasma Physics and Controlled Fusion 51 (2009).

[10] K. Budden, The Propagation of Radio Waves, vol. 596, Cambridge University Press, Cambridge, 1985.

[11] R.Z. Sagdeev, V.D. Shapiro, Pis'ma Zh. Eksp. Teor. Fiz. 17 (1973) 389.

[12] K.S. Golovanivsky, V.D. Dougar-Jabon, D.V. Reznikov, Physical Review E 52 (1995) 2969.

[13] K. Nagaoka, A. Okamoto, S. Yoshimura, M. Kono, M.Y. Tanaka, Physical Review Letters 89 (1992) 7.

[14] S. Yoshimura, A. Okamoto, M. Tanaka, Physical Review Letters 8 (2009) 11.

[15] D. Mascali, L. Celona, S. Gammino, R. Miracoli, G. Castro, N. Gambino, G. Ciavola, Nuclear Instruments and Methods A (2011), http://dx.doi.org/10.1016/j.nima. 2011.02.04.

[16] S. Tudisco et al. 653 (2011) 47-51.

[17] N. Gambino et al. LNS-INFN Report, 2009.

[18] G. Castro, et al., Review of Scientific Instruments 83 (2012) 02 B501.

[19] N.M. Bulgakova et al., Physical Review E, 62, 2000.

[20] H. Hora, Non Linear Plasma Dynamics at Laser Irradiation, Springer-Verlag, Berlin, 1979. 